\title{
Linfoma no Hodgkin primario en fémur: reporte de caso y revisión sistemática del protocolo diagnóstico en ortopedia
}

\section{Primary Non-Hodgkin Lymphoma in femur: case report and systematic review of the orthopedic's diagnosis protocol}

\author{
Óscar B. Terán-Maxínez ${ }^{1 *}$, Sergio Gómez Llata-García1 , Carlos A. Sánchez-Mojica ${ }^{1,2}$, \\ Verónica Durán-Pérez ${ }^{2}$ y Ana L. Espinosa-Cortes ${ }^{3}$
}

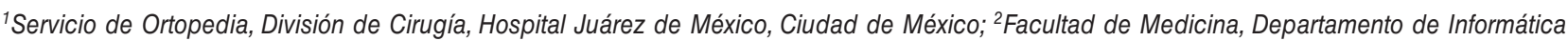
Biomédica, Universidad Nacional Autónoma de México, Ciudad de México; ${ }^{3}$ Facultad de Estudios Superiores Iztacala, Departamento de Docencia de Ciclos clínicos, Universidad Nacional Autónoma de México, Estado de México. México

\section{Resumen}

\begin{abstract}
Antecedentes: El linfoma no Hodgkin (LNH) es una neoplasia maligna que la Organización Mundial de la Salud reporta con gran incidencia y mortalidad en México. El objetivo del trabajo es realizar una revisión sistemática del protocolo diagnóstico del LNH primario de hueso en el servicio de ortopedia. Métodos y resultados: Se realizó una revisión sistemática en PubMed, OVID, EBSCO y Cochrane. La búsqueda inició con los términos MeSH en inglés: Primary bone lymphoma y relacionados (Non-Hodgkin, bony, osseous...). Se seleccionaron solo los artículos del LNH en población adulta que correspondieran a tumores primarios en hueso. De una búsqueda inicial de 31,570 artículos, 196 fueron seleccionados para lectura completa, se excluyeron 154 por no cumplir con los criterios. Conclusiones: Los protocolos enfatizan una historia clínica dirigida y estudios de imagen más biopsia diagnóstica con estudio histopatológico de la muestra. El tratamiento ortopédico sigue parámetros similares al LNH clásico, siendo las fracturas en terreno patológico la principal indicación de tratamiento ortopédico.
\end{abstract}

Palabras clave: Linfoma no Hodgkin. Linfoma primario en hueso. Ortopedia. Diagnóstico.

\section{Abstract}

Background: Non-Hodgkin's Lymphoma (NHL) is a malignant neoplasm that the World Health Organization reports with a high incidence and mortality in Mexico. The objective of the work is to carry out a systematic review of the diagnostic protocol of primary Non-Hodgkin's Lymphoma of bone in the orthopedic service. Methods and Results: A systematic review was carried out in PubMed, OVID, EBSCO and Cochrane. The search started with the MeSH terms in English: Primary Bone Lymphoma and related (Non-Hodgkin, Bony, osseous...). Only non-Hodgkin lymphoma articles in the adult population corresponding to primary bone tumors were selected. From an initial search of 31,570 articles, 196 were selected for full reading, 154 were excluded because they did not meet the criteria. Conclusions: The protocols emphasize a directed clinical history and imaging studies plus diagnostic biopsy with histopathological study of the sample. Orthopedic treatment follows parameters similar to classic NHL, with fractures in the pathological terrain being the main indication for orthopedic treatment.

Key words: Non-Hodgkin's lymphoma. Primary bone lymphoma. Orthopedics. Diagnosis.

Correspondencia:

*Óscar B. Terán-Maxínez

E-mail: oscar_1990_10@ hotmail.com
Disponible en internet: 13-09-2021 Rev Hosp Jua Mex. 2021;88(3):140-150

www.revistahospitaljuarez.com licencia CC BY-NC-ND (http://creativecommons.org/licenses/by-nc-nd/4.0/). 


\section{Introducción}

Los linfomas óseos primarios se pueden clasificar como linfoma Hodgkin (LH) y linfoma no Hodgkin (LNH). Ambos tipos de neoplasias tienen un comportamiento maligno y su presentación es rara, típicamente es una lesión solitaria que surge de la cavidad medular con dolor óseo localizado. La mayoría de los linfomas corresponden al tipo $\mathrm{LNH}$, mientras que el $\mathrm{LH}$ primario del hueso es extremadamente raro, por lo cual en este texto haremos referencia al linfoma óseo primario (LOP) como aquellos del tipo $\mathrm{LNH}^{1}$.

EI LOP se describe en la clasificación de tumores de tejido blando y hueso de la Organización Mundial de la Salud (OMS) como una neoplasia linfoide maligna que produce al menos una masa dentro del hueso, sin la participación de ganglios linfáticos suprarregionales u otros sitios extranodales. Esta definición fue interpretada más tarde por Messina, et al. para representar: a) una sola lesión ósea con o sin afectación de los ganglios linfáticos regionales o b) múltiples lesiones óseas, pero sin ganglios linfáticos o enfermedad visceral. La definición de LOP excluye los linfomas que se han diseminado desde los ganglios linfáticos o sitios extraganglionares y representan afectación esquelética secundaria ${ }^{2-4}$.

En los últimos reportes encontrados, el LNH representa el $7 \%$ de los tumores óseos malignos, menos del $1 \%$ de los linfomas malignos, y de ellos tan solo el $4.5 \%$ tienen una presentación extranodal. De este último grupo la presentación primaria en hueso representa solo del 3 al $5 \%$ de todos los linfomas en adultos. La incidencia del LOP es tan rara $(0.5 \%$ aproximadamente) que muchos aspectos de la enfermedad siguen sin resolverse ${ }^{1,5}$.

La mayoría de los casos se reportan con preponderancia del sexo masculino sobre el femenino, con una relación 1.2 a 1.8, con una edad de presentación entre los 30 y los 60 años y una incidencia máxima en la quinta o sexta década de la vida. La presentación pediátrica del linfoma primario en hueso es muy rara y la mayoría de los reportes la consideran una entidad clínica distinta por necesitar un enfoque de tratamiento diferente al de los adultos ${ }^{2,5-8}$.

El cuadro clínico que presentan los pacientes con LOP es muy variado y puede estar constituido por signos y síntomas leves que pueden persistir por largo tiempo antes de que el paciente decida buscar atención médica: dolor leve en la zona de afectación, edema, fiebre, pérdida de peso y sudoración nocturna (síntomas B), hasta un cuadro clínico de características más agresivas con dolor óseo que no calma con el reposo, masas palpables en el sitio primario (generalmente secundario a la extensión del tejido blando alrededor del hueso) $)^{6,9}$.

Los artículos más recientes mencionan que los principales sitios de afectación por LOP son los huesos largos de las extremidades y el esqueleto axial. El hueso reportado como principal sitio de aparición del LOP es el fémur, representando entre el 20 y el $30 \%$ de los pacientes con linfoma primario de hueso (PLB). Otros sitios comunes de presentación son la pelvis $(15 \%)$, la tibia (13\%), el húmero (12\%) y la columna vertebral $(9 \%)$, mientras que otros sitios representaron solo el 6\% de los casos s.9-11. $^{5}$.

\section{Material y métodos}

\section{Reporte de caso}

De acuerdo con la guía CARE (CAse REports), se documentaron los siguientes apartados: información del paciente, hallazgos clínicos relacionados con el padecimiento y encontrados en la exploración física, evaluación diagnóstica e intervención terapéutica realizada.

\section{Revisión sistemática}

La revisión realizada se apegó a lo establecido en la declaración PRISMA (Preferred Reporting Items for Systematic reviews and Meta-Analyses). Debido a la baja frecuencia de esta enfermedad se incluyeron todos los tipos de estudio, posteriormente se eligieron artículos publicados en inglés o español, desde el año 1969 a 2020, que correspondieran a tumores primarios en hueso en población adulta humana.

Las fuentes de información utilizadas para la revisión sistemática fueron PubMed, OVID, EBSCO y Cochrane. La búsqueda en cualquiera de las fuentes inició con los términos MeSH en inglés: primary bone lymphoma y relacionados (non-Hodgkin, bony, osseous,...). Posteriormente, se trasladó la información de título, autores, cita, fuente y resumen a una tabla. Se eliminaron los resultados que la patología que no correspondía a la estudiada, duplicados y los que no cumplieron los criterios de elegibilidad.

De los estudios seleccionados para la lectura del resumen, se descartaron los artículos que presentara LOP en un sitio diferente a fémur, los que no contaban con datos suficientes para el análisis del protocolo diagnóstico terapéutico en ortopedia y los que 
corresponden a LNH secundario o asociado a metástasis. El proceso de selección de artículos se describe en el algoritmo de la figura 1. Para completar la información disponible se consultó el tratamiento en guías de práctica clínica internacionales relacionadas con $\mathrm{LNH}$ o tumores primarios en hueso.

\section{Resultados}

\section{Reporte de caso}

El caso reportado en este estudio es el de una mujer adulta de 44 años, mexicana, tratada en el Hospital Juárez de México, originaria y residente del Estado de México, católica, ama de casa, con escolaridad de primaria incompleta.

\section{Motivo de consulta}

Refiere que su padecimiento inició a principio del mes de septiembre de 2017, al sufrir caída desde aproximadamente $50 \mathrm{~cm}$ de altura de vehículo detenido. Posteriormente, presentó dolor en muslo distal izquierdo con intensidad 5 de 10 en la escala visual analógica (EVA). Acudió a un "sobador", quien le solicitó una radiografía, sin embargo, no volvió a consultar a dicha persona. Quince días después sufrió otra caída, por lo cual acudió a un quiropráctico, quien le solicitó nuevamente una radiografía y le inició manejo farmacológico no especificado. Al no mostrar mejoría acudió a un facultativo particular, quien la refiere a nuestro servicio para valoración.

- Antecedentes heredofamiliares: refiere carga genética para diabetes mellitus.

- Antecedentes personales no patológicos: habita casa de materiales perdurables en medio urbano, menciona que en un municipio vecino al suyo se encuentra un depósito de residuos industriales, cohabita con una persona, su alimentación es mala en cantidad y calidad.

- Antecedentes personales patológicos: padece diabetes mellitus tipo II de ocho años de diagnóstico en tratamiento con insulina NPH (Neutral Protamine Hagedorn) $30 \mathrm{UI}-0-15 \mathrm{UI}$ en descontrol. Cuenta con el antecedente de cesárea hace 13 años sin complicaciones. Refiere tabaquismo positivo desde los 16 años, llegando a fumar 5 cigarrillos diarios por 10 años, actualmente suspendido. Menciona etilismo ocasional positivo.

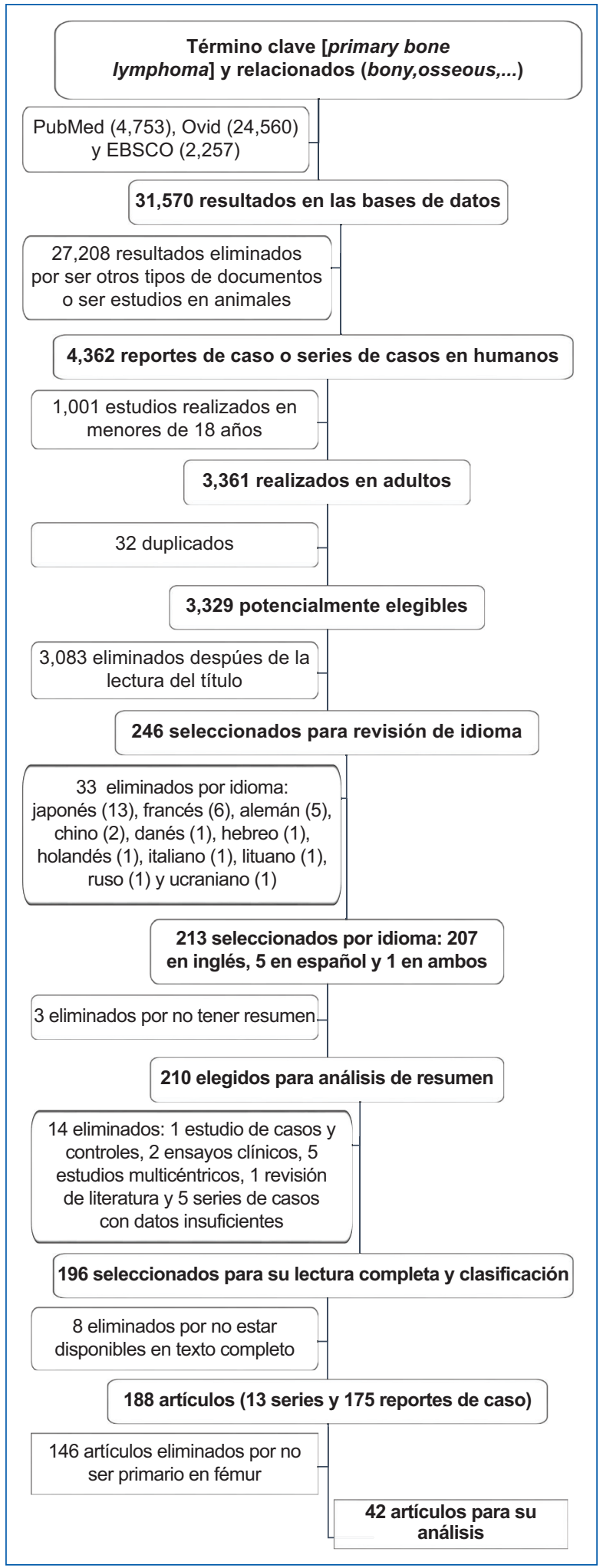

Figura 1. Algoritmo de selección de artículos. Se describe el proceso de selección de los artículos de acuerdo con los criterios de elegibilidad establecidos. 
- Exploración física: cráneo sin exostosis o hundimientos, con pupilas isocóricas, normorrefléxicas, narinas permeables. Cuello cilíndrico simétrico sin adenomegalias, con tráquea central móvil. Tórax con adecuados movimientos de amplexión, amplexación, con buena entrada y salida de aire, sin fenómenos exudativos agregados; los ruidos cardiacos de buen tono, frecuencia e intensidad. Abdomen blando depresible, timpánico a la percusión, no doloroso con perístasis presente normoactiva, se encuentra sin megalias, sin datos de irritación peritoneal. Extremidades torácicas íntegras, simétricas, con adecuados arcos de movilidad, fuerza muscular $5 / 5$ en escala de Daniel. Sin datos de compromiso neurovascular distal, sin cadenas ganglionares palpables. Extremidad pélvica izquierda íntegra, con aumento de volumen a nivel de tercio distal de muslo, con lesión sólida, fija a planos profundas, dolorosa a la palpación y a la flexoextensión de rodilla (Fig. 2). No se observa red venosa colateral, sin datos de compromiso neurovascular distal. Resto de la exploración física sin alteraciones.

- Interpretación radiográfica: proyección anteroposterior y lateral de fémur izquierdo en la que se observaron lesiones infiltrativas a nivel de tercio distal con destrucción de la cortical y reacción perióstica formando triángulo de Codman hacia la región metafisaria y ondulante e irregular a nivel de la diáfisis (Fig. 3). Se decidió el ingreso de la paciente para iniciar protocolo de estudio.

- En los estudios de laboratorio únicamente se encontraron alteraciones de los niveles de glucosa sérica, asociados al mal control de la diabetes mellitus tipo II.

- El 27 de octubre de 2017 se realizó gammagrafía ósea con tecnecio 90, en la cual se observó captación elevada de radiofármaco a nivel de bóveda craneana, tercio medio de fémur y región metaepifisaria ipsilateral. Se reportó «alta probabilidad de metástasis óseas" (Fig. 4).

- El 21 de noviembre de 2017 se realizó biopsia excisional de fémur distal izquierdo y se envió la muestra al servicio de patología para su estudio.

- El 6 de diciembre de 2017 se recibió el resultado del estudio histopatológico, que reportó: tejido óseo con infiltración por linfoma folicular grado II de la OMS (CD20, BCL2). Las reacciones de inmunohistoquímica reportaron: CD20 positivo, BCL-2 positivo, BCL-6 (+/-) y KI67: $60 \%$. Con estos resultados se confirmó el diagnóstico de linfoma folicular óseo probablemente primario de tipo no Hodgkin en fémur distal izquierdo.

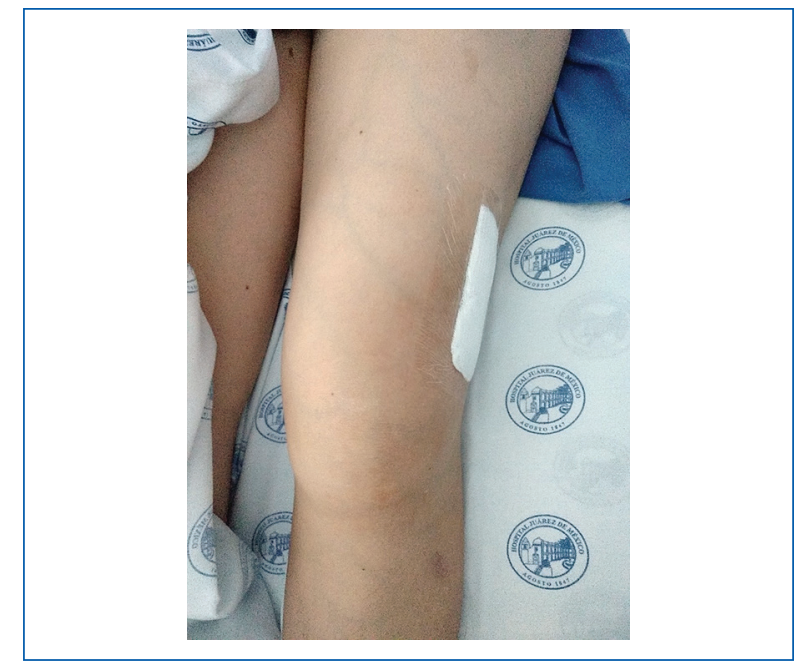

Figura 2. Fotografía de la extremidad pélvica izquierda de la paciente al comienzo del cuadro clínico. Se observa extremidad pélvica izquierda íntegra, con aumento de volumen a nivel de tercio distal de muslo, con lesión sólida, fija a planos profundos, dolorosa a la palpación y a la flexoextensión de rodilla. La paciente dio su consentimiento verbal para el uso de la imagen.

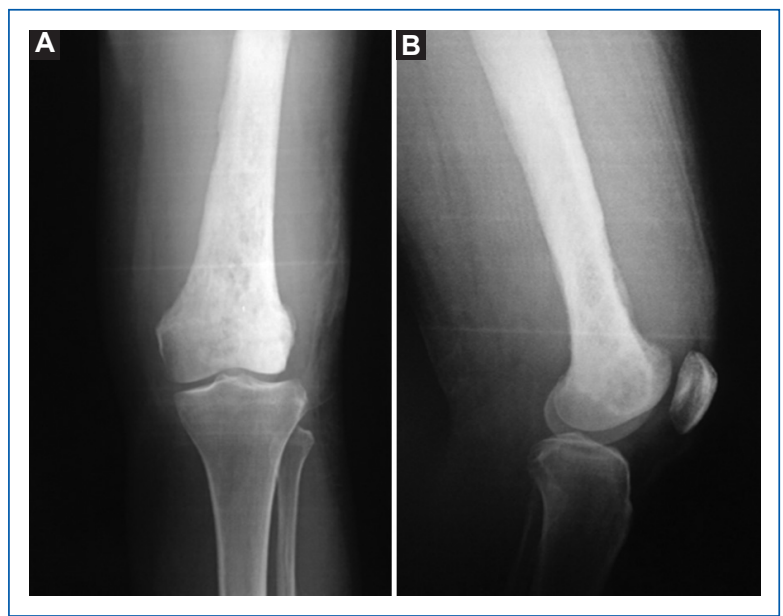

Figura 3. Serie radiográfica de fémur izquierdo de la paciente. Proyección anteroposterior (A) y lateral (B) de fémur izquierdo en la que se observaron lesiones infiltrativas a nivel de tercio distal con destrucción de la cortical y reacción perióstica formando triángulo de Codman hacia la región metafisaria y ondulante e irregular a nivel de la diáfisis.

- Se envía interconsulta al servicio de hematología, quienes decidieron cambio de servicio para continuar con protocolo de diagnóstico y tratamiento. Ya a su cargo, se realizó tomografía computarizada (TC) toracoabdominal que descartó afectación ganglionar 


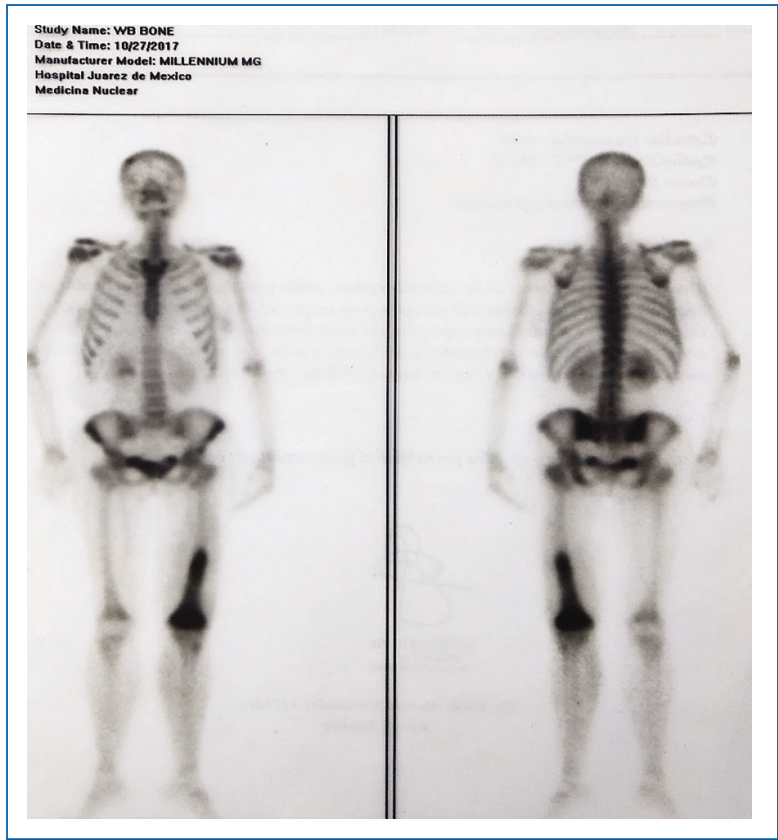

Figura 4. Gammagrafía ósea con tecnecio 90 de la paciente. Se observó captación elevada de radiofármaco a nivel de bóveda craneana, tercio medio de fémur y región metaepifisaria ipsilateral. Se reportó "alta probabilidad de metástasis óseas».

a distancia. El 14 de diciembre de 2017 se inició el primer ciclo de quimioterapia con esquema $\mathrm{CHOP}$ (ciclofosfamida, clorhidrato de doxorubicina (hidroxidaunorubicina), sulfato de vincristina [Oncovin] y prednisona).

\section{Revisión sistemática}

De la búsqueda inicial de 31,570 artículos, 196 fueron seleccionados para su lectura completa, de ellos se excluyeron 154 por no cumplir con los criterios de elegibilidad. Por las características de los 42 estudios seleccionados no se realizó un metaanálisis (Fig. 1), pero con la intención de obtener un consenso de las recomendaciones para el manejo del LOP en fémur se elaboró una tabla comparativa para el análisis sistemático de la información aportada por cada estudio ${ }^{12-53}$.

De los 42 artículos incluidos para el análisis 34 corresponden a reportes de casos, 6 son series de caso y 2 reporte de caso y revisión bibliográfica sobre LOP. En la tabla 1 se mencionan las características de cada estudio (autor, año, referencia, país, tipo de estudio) ${ }^{12-53}$.

Las características demográficas y clínicas de los pacientes se reportan en la tabla 2. Se analizó la distribución por sexo de casos de LNH y se observó un
Tabla 1. Características y principales conclusiones de los estudios analizados

\begin{tabular}{|c|c|c|}
\hline Autor & País & Tipo de estudio \\
\hline Abdelwahab, et al., 2007 & EE.UU. & Reporte de caso \\
\hline Agarwal, et al., 2001 & India & Reporte de caso \\
\hline Anyfanti P, et al., 2015 & Grecia & Reporte de caso \\
\hline Burmeister BH, et al., 1989 & $\begin{array}{l}\text { Nueva } \\
\text { Zelanda }\end{array}$ & Reporte de caso \\
\hline Campbell SE, et al., 2003 & EE.UU. & Reporte de caso \\
\hline Christie DR, et al., 1996 & Australia & Reporte de caso \\
\hline Cowie F, et al., 1991 & Inglaterra & Reporte de caso \\
\hline Eustace S, et al., 1995 & EE.UU. & Reporte de caso \\
\hline Gebert C, et al., 2005 & Alemania & Reporte de caso \\
\hline Goshen E, et al., 1997 & Israel & Reporte de caso \\
\hline Hatori M, et al., 1997 & Japón & Reporte de caso \\
\hline Hayashi M, et al., 2005 & Japón & Reporte de caso \\
\hline Hermann G, et al., 1999 & EE.UU. & Reporte de caso \\
\hline Hsieh TC, et al., 2007 & Taiwán & Reporte de caso \\
\hline Hu JY, et al., 2018 & China & Reporte de caso \\
\hline Ishiguro K, et al., 2016 & Japón & Reporte de caso \\
\hline Iwahara Y, et al., 2017 & Japón & Reporte de caso \\
\hline Jawa A, et al., 2006 & EE.UU. & Reporte de caso \\
\hline Lacor P, et al., 1990 & Bélgica & Reporte de caso \\
\hline Lamichhane A, et al., 2013 & Nepal & Reporte de caso \\
\hline McCammon J, et al., 2014 & Canadá & Reporte de caso \\
\hline Misgeld E, et al., 2003 & Alemania & $\begin{array}{l}\text { Reporte de caso y } \\
\text { revisión sistémica }\end{array}$ \\
\hline Pinheiro RF, et al., 2009 & Brasil & Reporte de caso \\
\hline Riaz IP, et al., 2017 & Pakistán & Reporte de caso \\
\hline $\begin{array}{l}\text { Romero-Rojas AE, et al., } \\
2014\end{array}$ & Colombia & Reporte de caso \\
\hline Roy S, et al., 2005 & Australia & Reporte de caso \\
\hline Sato H, et al., 1993 & Japón & Reporte de caso \\
\hline Schmidt A, et al., 1994 & Alemania & Reporte de caso \\
\hline Schulze T, et al., 2009 & Alemania & Reporte de caso \\
\hline Singh T, et al., 2010 & India & $\begin{array}{l}\text { Reporte de caso y } \\
\text { revisión sistemática }\end{array}$ \\
\hline Singh $\mathrm{T}$, et al (2011 & India & Reporte de caso \\
\hline Sipsas NV, et al., 2002 & Grecia & Reporte de caso \\
\hline Stephens GC, et al., $1994^{44}$ & EE.UU. & Reporte de caso \\
\hline
\end{tabular}


Tabla 1. (Continuación)

\begin{tabular}{|l|l|l|}
\hline Autor & País & Tipo de estudio \\
\hline Sugisawa N, et al., 2006 & Tokio & Reporte de caso \\
\hline Visser J, et al., 201246 & $\begin{array}{l}\text { Países } \\
\text { bajos }\end{array}$ & Reporte de caso \\
\hline Xie XT, et al., 201147 & China & Reporte de caso \\
\hline Yamamoto S, et al., 2019 & Japón & Reporte de caso \\
\hline Böhm P, et al., 200149 & Alemania & Reporte de caso \\
\hline Lucraft HH, 199153 & $\begin{array}{l}\text { Reino } \\
\text { Unido }\end{array}$ & Reporte de caso \\
\hline Ngan H, et al., 197550 & $\begin{array}{l}\text { Reino } \\
\text { Unido }\end{array}$ & Reporte de caso \\
\hline $\begin{array}{l}\text { Radaszkiewicz T, et al., } \\
\text { 198851 }\end{array}$ & Alemania & Reporte de caso \\
\hline Sweet, et al., 198152 & EE.UU. & Reporte de caso \\
\hline
\end{tabular}

claro predominio en hombres, con una razón proporción mujer:hombre de 1:1.7. También se analizó la edad de presentación, que fue de 45.4 años en promedio, en un rango de 19 a 92 años. El cuadro clínico más común reportado presenta el dolor en el sitio afectado como primer dato clínico, seguido de inflamación y en tercer lugar masa palpable, síntomas como artralgias y compresión medular, entre otros, se presentaron de manera inconsistente. La incidencia de LOP en fémur se presenta principalmente en la sexta década de la vida $^{12-53}$.

Los diferentes abordajes diagnósticos terapéuticos reportados incluyen siempre un estudio de imagen, aunque difieren en el estudio utilizado para el diagnóstico; en la figura 5 se observa la frecuencia en que se solicitaron los diferentes tipos de estudios de imagen utilizados en los protocolos reportados. Podemos ver que, como era de esperarse, el primer estudio de imagen solicitado en el $100 \%$ de los casos de pacientes con LOP es la radiografía, seguido de la resonancia magnética (RM) y en tercer lugar la $\mathrm{TC}^{12-53}$.

En general se reportan tres tipos de patrones radiográficos: a) patrón lítico destructivo, es el más común y a su vez algunos reportes lo subclasifican en patrón permeativo y patrón en sacabocado; b) patrón blástico esclerótico, es raro y algunas veces se acompaña de lesiones líticas y escleróticas para convertirse en un patrón al que llaman «mixto», o c) hallazgos sutiles o "casi normales" a pesar de que los pacientes presentan síntomas clínicos, si bien esos son los patrones más comúnmente reportados, hasta en un 16\% de los

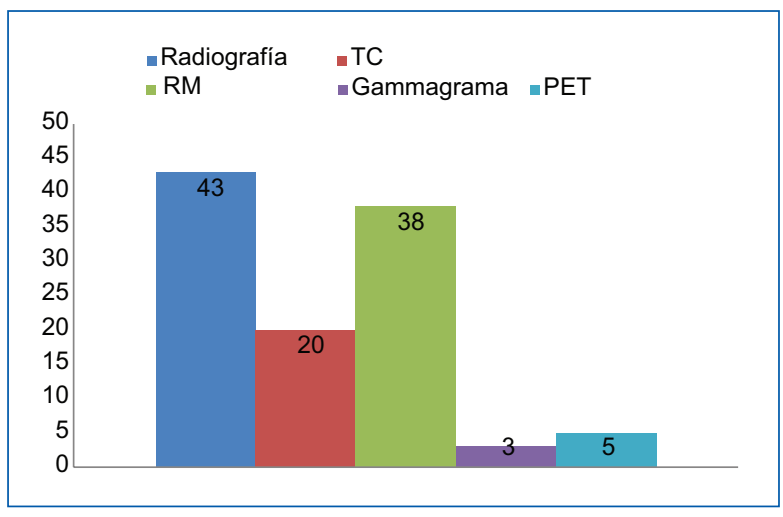

Figura 5. Estudios de imagen utilizados en los reportes consultados.

TC: tomografía computarizada; RM: resonancia magnética; PET: tomografía por emisión de positrones.

casos la imagen radiográfica no concuerda con los patrones previamente descritos.

El segundo estudio más utilizado en el protocolo diagnóstico de los pacientes con LOP es la RM, aunque la aparición del LOP en este estudio es muy variable. La extensión de los tejidos blandos se identifica con frecuencia y suele ser homogéneamente isointensa con músculo en las imágenes ponderadas en T1 y alta en las imágenes ponderadas en T2; la mayoría de los pacientes tienen hueso cortical de apariencia anormal y la intensidad de la señal de la afectación de la médula ósea es heterogénea en aproximadamente la mitad de los casos, en comparación con el componente de tejidos blandos, y es alta o intermedia en las imágenes ponderadas en T2 en la mayoría de los casos.

En la TC el principal patrón reportado es el osteolítico, en aproximadamente el $80 \%$ de las TC, aunque en muchos casos este patrón se reporta asociado con otros patrones diferentes a este, como lo son el osteoesclerótico o mixto ${ }^{12-53}$.

Por último, el uso del gammagrama se reporta en el $12 \%$ de los artículos y de la tomografía por emisión de positrones (PET) tan solo en el 7\%. Como principales hallazgos en el gammagrama se encontraron la actividad metabólica y osteoblástica activa y la lesión extranodal. Por otro lado, la enfermedad ósea marcada y el involucro de linfonodos son los principales hallazgos reportados por la PET.

Los artículos que presentan casos de LOP en hueso mencionan que, tras una sospecha por clínica y estudio de imagen, se realizaron estudios de anatomía patológica; en la tabla 3 se reportan los principales 
Tabla 2. Características demográficas y clínicas de los casos

\begin{tabular}{|c|c|c|c|}
\hline Estudio & Sexo & Edad & Presentación clínica \\
\hline Abdelwahab, et al., 2007 & H & 27 & Dolor, inflamación y masa palpable \\
\hline Agarwal, et al., 2001 & H & 72 & Dolor, inflamación, debilidad y masa palpable \\
\hline Anyfanti P, et al., 2015 & H & 50 & Fiebre, dolor e inflamación de espalda baja \\
\hline Burmeister BH, et al., 1989 & M & 43 & Dolor, masa palpable e inflamación \\
\hline Campbell SE, et al., 2003 & M & 20 & Dolor, debilidad, masa palpable e inflamación \\
\hline Christie DR, et al., 1996 & $\mathrm{H}: 10 \mathrm{M}: 7$ & $28-92$ & Dolor e inflamación \\
\hline Cowie F, et al., 1991 & M & 19 & Dolor, debilidad, inflamación, pérdida de peso, masa palpable \\
\hline Eustace S, et al., 1995 & H & 63 & Dolor de pelvis y pérdida de peso \\
\hline Gebert C, et al., 2005 & H & 21 & Dolor, inflamación de rodilla izquierda \\
\hline Goshen E, et al., 1997 & H & 24 & Dolor, inflamación de fémur izquierdo \\
\hline Hatori M, et al., 1997 & H & 41 & Dolor de cadera y rodilla izquierdos \\
\hline Hayashi M, et al., 2005 & $\mathrm{H}: 2 \mathrm{M}: 3$ & $50-71$ & Dolor y pérdida de la función de pelvis, fémur \\
\hline Hermann G, et al., 1999 & H & 66 & Dolor, inflamación de fémur proximal derecho \\
\hline Hsieh TC, et al., 2007 & M & 30 & Dolor de cadera derecha \\
\hline Hu JY, et al., 2018 & M & 65 & Dolor de ambos muslos \\
\hline Ishiguro K, et al., 2016 & M & 63 & Dolor, debilidad e inflamación \\
\hline Iwahara Y, et al., 2017 & H & 60 & Dolor e inflamación de fémur distal derecho \\
\hline Jawa A, et al., 2006 & H & 33 & Dolor, debilidad de rodilla derecha \\
\hline Lacor P, et al., 1990 & H & 45 & Dolor de cadera izquierda \\
\hline Lamichhane A, et al., 2013 & H & 45 & Dolor y masa palpable \\
\hline McCammon J, et al., 2014 & H & 80 & Dolor en muslo izquierdo e inflamación \\
\hline Misgeld E, et al., 2003 & H & 30 & Dolor de pierna derecha con información \\
\hline Pinheiro RF, et al., 2009 & H & 63 & Dolor, inflamación y pérdida de peso \\
\hline Riaz IP, et al., 2017 & H & 71 & Dolor, debilidad y pérdida de peso \\
\hline Romero-Rojas AE, et al., 2014 & H & 27 & Dolor de rodilla derecha con debilidad \\
\hline Roy S, et al., 2005 & H & 20 & Masa palpable en rodilla derecha \\
\hline Sato H, et al., 1993 & H & 84 & Dolor, inflamación y claudicación \\
\hline Schmidt A, et al., 1994 & H & 25 & Dolor e inflamación \\
\hline Schulze T, et al., 2009 & H & 63 & Dolor e inflamación \\
\hline Singh T, et al., 2010 & H & 44 & Dolor de muslo izquierdo \\
\hline Singh T, et al (2011 & H & 35 & Dolor de rodilla izquierda \\
\hline Sipsas NV, et al., 2002 & H & 38 & Inflamación y masa en cadera derecha \\
\hline Stephens GC, et al., $1994^{44}$ & H & 50 & Dolor en muslo izquierdo \\
\hline Sugisawa N, et al., 200645 & H & 70 & Dolor de rodilla derecha con masa palpable \\
\hline Visser J, et al., $2012^{46}$ & H & 69 & Dolor de rodilla izquierda con inflamación \\
\hline Xie XT, et al., $2011^{47}$ & H & 47 & Dolor en muslo derecho con inflamación y deambulación \\
\hline Yamamoto S, et al., $2019^{23}$ & H & 56 & Dolor en rodilla izquierda \\
\hline Böhm P, et al., $2001^{49}$ & H: 1 M: 1 & $33-38$ & Dolor en miembro pélvico \\
\hline Lucraft HH, $1991^{53}$ & H: 7 M: 6 & $28-86$ & $\begin{array}{l}\text { Dolor en costillas, pelvis } 3 \text {, fémur } 3 \text {, maxilar, húmero, clavícula, } \\
\text { escápula, tibia, mandíbula 2, columna lumbar }\end{array}$ \\
\hline Ngan H, et al., $1975^{50}$ & H: 2 M: 3 & $20-57$ & $\begin{array}{l}\text { Dolor en muslo derecho, dolor en muslo izquierdo, dolor en pelvis, dolor } \\
\text { en nalga izquierda, dolor en cadera derecha }\end{array}$ \\
\hline Radaszkiewicz T, et al., $1988^{51}$ & H: 1 M: 2 & $44-66$ & Dolor y edema de cresta iliaca, dolor de ingle izquierda, dolor lumbar \\
\hline Sweet, et al., $1981^{52}$ & H: 5 M: 3 & $10-74$ & Dolor en escápula, fémur 4, maxilar, vertebral, tibia y escápula \\
\hline
\end{tabular}


Tabla 3. Estudios de anatomía patológica

\begin{tabular}{|c|c|c|c|}
\hline Estudio & Biopsia diagnóstica & Inmunohistoquímica & Diagnóstico \\
\hline Abdelwahab, et al., 2007 & Biopsia abierta & & DLBCL \\
\hline Agarwal, et al., 2001 & Biopsia por aguja & CD3, CD45 & DLBCL \\
\hline Anyfanti P, et al., 2015 & Biopsia por aguja & CD10, CD20 & DLBCL \\
\hline Burmeister BH, et al., 1989 & Biopsia abierta & & DLBCL \\
\hline Campbell SE, et al., 2003 & Biopsia por aguja & CD20, CD45 & DLBCL \\
\hline Christie DR, et al., 1996 & Biopsia por aguja & & DLBCL \\
\hline Cowie F, et al., 1991 & Biopsia abierta & & Linfoma de Hodgkin óseo primario \\
\hline Eustace S, et al., 1995 & Biopsia por aguja & & Linfoma óseo multifocal primario hueso \\
\hline Gebert C, et al., 2005 & Biopsia abierta & & Linfoma óseo multifocal primario hueso \\
\hline Goshen E, et al., 1997 & Biopsia abierta & & DLBCL \\
\hline Hatori M, et al., 1997 & Biopsia abierta & $\begin{array}{l}\text { CD5, CD10, CD19, CD22, CD1, } \\
\text { CD2, CD3, CD4, CD7, CD8 }\end{array}$ & DLBCL \\
\hline Hayashi M, et al., 2005 & Biopsia abierta & & DLBCL \\
\hline Hermann G, et al., 1999 & Biopsia abierta & & DLBCL \\
\hline Hsieh TC, et al., 2007 & Biopsia por aguja & & Linfoma óseo primario \\
\hline Hu JY, et al., 2018 & Biopsia abierta & CD20 & DLBCL \\
\hline Ishiguro K, et al., 2016 & Biopsia abierta & & DLBCL \\
\hline Iwahara Y, et al., 2017 & Biopsia abierta & $\mathrm{CD} 3, \mathrm{CD} 79 \mathrm{a}$ & DLBCL \\
\hline Jawa A, et al., 2006 & Biopsia abierta & CD10, CD20, bcl-2 & DLBCL \\
\hline Lacor P, et al., 1990 & Biopsia abierta & & DLBCL \\
\hline Lamichhane A, et al., 2013 & Biopsia incisional & & DLBCL \\
\hline McCammon J, et al., 2014 & Biopsia abierta & & DLBCL \\
\hline Misgeld E, et al., 2003 & Biopsia abierta & & DLBCL \\
\hline Pinheiro RF, et al., 2009 & Biopsia abierta & CD20 & DLBCL \\
\hline Riaz IP, et al., 2017 & Biopsia guiada por TC & & DLBCL \\
\hline Romero-Rojas AE, et al., 2014 & Biopsia abierta & CD20+DIM, CD79a+, BOB1 & DLBCL \\
\hline Roy S, et al., 2005 & Biopsia abierta & & DLBCL \\
\hline Sato H, et al., 1993 & Biopsia abierta & CD20, CD45 & DLBCL \\
\hline Schmidt A, et al., 1994 & Biopsia guiada por TC & CD20, CD45 & DLBCL \\
\hline Schulze T, et al., 2009 & Biopsia guiada por TC & & \\
\hline Singh T, et al., 2010 & Biopsia abierta & CD20, CD79a & $\mathrm{ALCL}$ \\
\hline Singh T, et al (2011 & Biopsia abierta & & DLBCL \\
\hline Sipsas NV, et al., 2002 & Biopsia excisional & CD4 & DLBCL \\
\hline Stephens GC, et al., $1994^{44}$ & Biopsia abierta & CD20, UCHL-1, CD43 & DLBCL \\
\hline Sugisawa $N$, et al., $20066^{45}$ & Biopsia abierta & $\mathrm{CD} 5, \mathrm{CD} 20, \mathrm{CD} 79 \mathrm{a}$ & DLBCL \\
\hline Visser J, et al., $2012^{46}$ & Biopsia abierta & $\mathrm{CD} 20$ & DLBCL \\
\hline Xie XT, et al., $2011^{47}$ & Biopsia abierta & & DLBCL \\
\hline Yamamoto S, et al., $2019^{23}$ & Biopsia por aguja & CD20, CD79a, MUM-1 & DLBCL \\
\hline Böhm P, et al., $2001^{49}$ & Biopsia por aguja & $\mathrm{CD} 20$ & DLBCL \\
\hline Lucraft HH, $1991^{53}$ & Biopsia abierta & & DLBCL \\
\hline $\mathrm{Ngan} \mathrm{H}$, et al., $1975^{50}$ & Biopsia abierta & & DLBCL \\
\hline Radaszkiewicz T, et al., $1988^{51}$ & Biopsia por aguja & & DLBCL \\
\hline Sweet, et al., $1981^{52}$ & Biopsia abierta & & Linfoma primario de hueso \\
\hline
\end{tabular}

ALCL: linfoma anaplásico de células grandes; DLBCL: diffuse large B-cell lymphoma; TC: tomografía computarizada. 


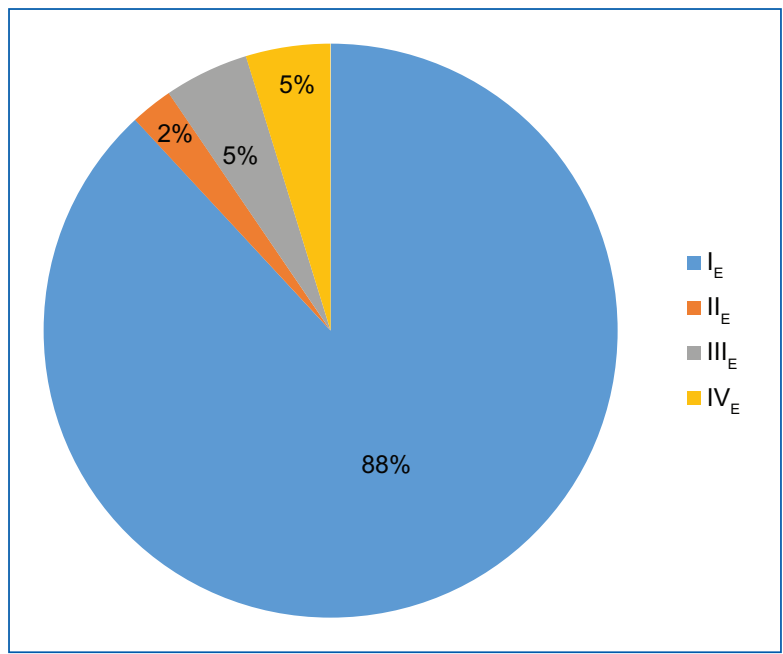

Figura 6. Casos de acuerdo con el sistema de estadificación de Ann Arbor.

estudios y sus resultados diagnósticos. El diagnóstico se realizó en la mayoría de los casos mediante evaluación histopatológica con biopsia abierta, aunque también están reportados la realización de biopsia con aguja y guiada con TC. En el reporte de inmunohistoquímica encontramos que los principales marcadores de esta patología son: CD20, CD45, CD10 y CD3. Para clasificar los tumores se utilizó la clasificación actual de la OMS de los tumores de los tejidos hematopoyéticos y linfoides, donde se describe las diferentes categorías de las neoplasias linfoides. Histopatológicamente, los estudios reportaron que la mayoría de los casos con linfoma primario no Hodgkin (LPNH) fueron del subtipo DLBCL ${ }^{12-53}$.

En la figura 6 se presenta el número de casos por estudio de acuerdo con el sistema de estadificación de Ann Arbor, donde observamos que el estadio más común de presentación al diagnóstico corresponde al $\mathrm{I}_{E}$, seguido por el III $\mathrm{E}_{\mathrm{E}} \mathrm{IV}_{\mathrm{E}}^{12-53}$.

\section{Conclusiones}

El abordaje inicial que debemos tener en un paciente en quien sospechamos LOP requiere una historia clínica completa, un examen físico con énfasis en el sitio involucrado y solicitud de radiografías simples del sitio afectado. Debemos considerar el LOP entre los diagnósticos diferenciales en un paciente adulto joven donde se observe en la radiografía una lesión solitaria en las metáfisis y epífisis de huesos largos con características lítica con patrón en sacabocado y una reacción perióstica agresiva. Los adultos mayores se ven mucho más afectados por la enfermedad metastásica o el mieloma que por el linfoma primario del hueso y aunque el patrón osteolítico en sacabocado y la reacción perióstica agresiva no son tan comunes, pueden ayudar a sugerir un linfoma primario de hueso en este grupo de edad.

La realización de este estudio permitió conocer que en aproximadamente el $50 \%$ de los pacientes con LOP se utiliza la RM. Nosotros recomendamos plenamente su uso de manera sistemática en el abordaje del paciente con LOP, ya que nos permite demostrar el reemplazo de la medula ósea, investigar la presencia de masas en tejido blando y demostrar de manera temprana la erosión cortical. Este último hallazgo es de especial interés, ya que la mayoría de los casos reportan una destrucción cortical sorprendentemente pequeña; entre las explicaciones propuestas para este suceso es que las células del LOP reaccionan ante las citocinas (interleucina [IL] 1, IL-6 y factor de necrosis tumoral) para estimular la activación de los osteoclastos y la producción de canales tumorales a través de la corteza, permitiendo así el escape del linfoma desde la cavidad intramedular hacia los tejidos blandos sin una destrucción cortical extensa. Debemos mencionar que esto último se puede observar también en la TC, sin embargo, la RM ha demostrado su detección de forma más temprana comparada con la TC.

Existe controversia sobre el estudio más sensible para el diagnóstico del LOP. Algunos artículos colocan a la RM como dicho estudio, pero también hay reportes que afirman que la gammagrafía con tecnecio es más sensible y específica; sin embargo, en los reportes analizados vemos que el uso del gammagrama no es algo claro ni sistemático en el estudio de los pacientes con sospecha de LOP.

Debemos hacer una mención especial a la PET, ya que, si bien la gammagrafía ósea refleja la actividad tumoral osteoblástica, la PET permite la caracterización de la actividad metabólica dentro del tumor en comparación con el tejido normal y es útil en la estadificación del linfoma, incluso en sitios extraganglionares como lo es el LOP. Otro uso importante reportado de la PET es su utilización para el seguimiento de los pacientes con LOP, ya que permite diferenciar entre el LOP persistente y la cicatrización ósea, diferencia no valorable en la gammagrafía.

Si bien se recomienda el uso de un examen de PET-TC integrada del tórax, el abdomen y la pelvis para determinar el grado de afectación de los ganglios linfáticos y la presencia de enfermedad a distancia, observamos que no es un determinante en el 
estudio del paciente, es curioso mencionar que existe un mayor reporte del uso de la PET que del gammagrama.

Si bien ya hemos logrado establecer hasta el momento un panorama de los datos imagenológicos del paciente con sospecha de LOP, su diagnóstico no es tan simple, ya que hay diversas presentaciones clínicas y radiológicas, y un amplio espectro de características histológicas. Por lo anterior, el gold standard para el diagnóstico del LPNH es mediante estudio histopatológico de las muestras obtenidas por biopsia percutánea o abierta. Histopatológicamente, los estudios reportan que la principal estirpe histológica de los LPNH en fémur fueron linfoma difuso de células $B$ grandes (diffuse large B-cell lymphoma, DLBCL), en segundo lugar los linfomas cutáneos de células $\mathrm{T}$ y linfoma de células $B$ inclasificable con características intermedias entre DLBCL y el LH clásico. El caso reportado en este trabajo es el primero en tener una estirpe folicular en la bibliografía reportada en los últimos 10 años.

\section{Conflicto de intereses}

Los autores declaran no tener conflicto de intereses alguno.

\section{Financiamiento}

La presente investigación no ha recibido ninguna beca específica de agencias de los sectores públicos, comercial, o sin ánimo de lucro.

\section{Responsabilidades éticas}

Protección de personas y animales. Los autores declaran que los procedimientos seguidos se conformaron a las normas éticas del comité de experimentación humana responsable y de acuerdo con la Asociación Médica Mundial y la Declaración de Helsinki.

Confidencialidad de los datos. Los autores declaran que han seguido los protocolos de su centro de trabajo sobre la publicación de datos de pacientes.

Derecho a la privacidad y consentimiento informado. Los autores han obtenido el consentimiento informado de los pacientes y/o sujetos referidos en el artículo. Este documento obra en poder del autor de correspondencia.

\section{Bibliografía}

1. Sánchez Mojica CA, Durán Pérez V, Sánchez L I, Sánchez Mojica LE, Ramírez CJG. Linfoma no Hodgkin primario en fémur: Reporte de caso y revisión sistemática del tratamiento ortopédico. Conferencia. XXX Congreso Mexicano de Ortopedia, FEMECOT. Hermosillo, octubre de 2019.

2. Chisholm KM, Ohgami RS, Tan B, Hasserjian RP, Weinberg OK. Primary lymphoma of bone in the pediatric and young adult population. Hum Pathol. 2017;60:1-10.

3. Campo E, Swerdlow SH, Harris NL, Pileri S, Stein H, Jaffe ES. The 2008 WHO classification of lymphoid neoplasms and beyond: Evolving concepts and practical applications. Blood. 2011;117(19):5019-32.

4. Swerdlow SH, Campo E, Pileri SA, Harris NL, Stein H, Siebert R, et al. The 2016 revision of the World Health Organization classification of lymphoid neoplasms. Blood J. 2017;127(20):453-62.

5. Jing-Yu H, Dan $Y$, Yao-Hui W. Primary non-Hodgkin lymphoma of the right femur and subsequent metastasis to the left femur: $\mathrm{A}$ case report and literature review. Oncol Lett. 2018;15:4427-31.

6. Mulligan ME, McRae GA. Lymphoma distinct. Most. 1999;(December):1691-7

7. Cai L, Stauder MC, Zhang YJ, Poortmans P, Li YX, Constantinou N, et al. Early-stage primary bone lymphoma: $A$ retrospective, multicenter Rare Cancer Network (RCN) study. Int $J$ Radiat Oncol Biol Phys. 2012;83(1):284-91

8. Jawad MU, Schneiderbauer MM, Min ES, Cheung MC, Koniaris LG, Scully SP. Primary lymphoma of bone in adult patients. Cancer. 2010;116(4):871-9.

9. Hoppe R, Illidge T, Specht L, Vogelius I, Yahalom J. Comment on: "Clinical Features, Management, and Prognosis of an International Series of 161 Patients With Limited-Stage Diffuse Large B-Cell Lymphoma of the Bone (the IELSG-14 Study)." Oncologist. 2014;19(12):1289.

10. Beal K, Allen L, Yahalom J. Primary bone lymphoma: Treatment results and prognostic factors with long-term follow-up of 82 patients. Cancer. 2006;106(12):2652-6.

11. Wu H, Zhang L, Shao H, Sokol L, Sotomayor E, Letson D, et al. Prognostic significance of soft tissue extension, International Prognostic Index, and multifocality in primary bone lymphoma: A single institutional experience. Br J Haematol. 2014;166(1):60-8.

12. Abdelwahab IF, Hoch B, Hermann G, Bianchi S, Klein MJ, Springfield DS. Primary periosteal lymphoma - Rare and unusual. Skeletal Radiol. 2007;36(4):335-9.

13. Agarwal V, Pal L, Aggarwal A, Misra R. Primary patellar T cell lymphoma: An unusual cause of monoarthritis. J Rheumatol. 2001;28(4):876-7.

14. Anyfanti $P$, Gouridou E, Tsinaridis A, Bekiaropoulos D, Argyropoulos E, Sousos N, et al. A treacherous case of primary non-Hodgkin lymphoma of the bone: Appearances can be deceptive. Scott Med J. 2015;60(3):9-13.

15. Burmeister $\mathrm{BH}$, Benjamin CS. Primary non-Hodgkins lymphoma of bone (case report). Australas Radiol. 1989;33(2):165-7.

16. Campbell SE, Filzen TW, Bezzant SM, Beall DP, Burton MP, Sanders TG, et al. Primary periosteal lymphoma: An unusual presentation of non-Hodgkin's lymphoma with radiographic, MR imaging, and pathologic correlation. Skeletal Radiol. 2003;32(4):231-5.

17. Christie D, Cahill S. BM. Primary bone lymphoma (Osteolymphoma). Australas Radiol. 1996:40:319-23.

18. Cowie F, Benghiat A, Holgate C. Case report primary Hodgkin's disease of bone. Clin Oncol. 1991;3(4):233-5.

19. Eustace S, O'Regan R, Graham D, Carney D. Primary multifocal skeletal Hodgkin's disease confined to bone. Skeletal Radiol. 1995;24(1):61-3.

20. Gebert C, Hardes J, Ahrens H, Buerger H, Winkelmann W, Gosheger G. Primary multifocal osseous Hodgkin disease: A case report and review of the literature. J Cancer Res Clin Oncol. 2005;131(3):163-8.

21. Goshen E, Shwartz A, Salai M, Apter S, Zwas S. Congruent uptake in bone and gallium scans in primary large-cell lymphoma of bone. Semin Nucl Med. 1997:27(1):75-8.

22. Hatori M, Ichinohasama R, Myers J, Schiller A, Kokubun S. Flow cytometrical and genotypic analysis of primary non-Hodgkin's lymphoma of bone. Pathol Res Pract. 1997;193(8):557-64.

23. Hayashi M, Tsuchiya $H$, Yamamoto $N$, Karita M, Shirai T, Nishida H, et al. Caffeine-potentiated chemotherapy for metastatic carcinoma and lymphoma of bone and soft tissue. Anticancer Res. 2005;25(3 C):2399-405.

24. Hermann G, Abdelwahab IF, Capozzi J, Springfield D, Klein MJ. Primary non-Hodgkin lymphoma of bone: Unusual manifestation of lymphoproliferative disease following liver transplantation. Skeletal Radiol. 1999;28(3):175-7.

25. Hsieh TC, Kao CH, Yen KY, Sun SS. Osteomyelitis-mimicking primary bone lymphoma at hip prosthetic site. Clin Nucl Med. 2007;32(7):543-4.

26. $\mathrm{Hu}$ JY, Yu D, Wu YH. Primary non-hodgkin lymphoma of the right femur and subsequent metastasis to the left femur: $A$ case report and literature review. Oncol Lett. 2018;15(4):4427-31.

27. Ishiguro $K$, Hayashi $T$, Aoki $Y$, Murakami $R$, Ikeda $H$, Ishida $T$, Othe iatrogenic immunodeficiency-associated lymphoproliferative disorder presenting as primary bone lymphoma in a patient with rheumatoid arthritis. Intern Med. 2016;55(16):2259-64. 
28. Iwahara Y, Shinohara T, Naruse K, Komatsu Y. Non-Hodgkin's lymphoma involving a femur bone and bilateral adrenal glands alone with adrena insufficiency. BMJ Case Rep. 2017;2017(C):2016-8.

29. Jawa $A$, Lieberman AE, Alexieva $C C N$, Jupiter JB. Primary intra-articular non-Hodgkin's lymphoma of the elbow. J Bone Joint Surg Am. 2006;88(12):2730-4

30. Lacor P, Cocquyt V, Schots R, Mathijs R, van Camp B. Malignant lymphoma of the bone. Acta Clin Belg. 1990;45(6):386-93.

31. Lamichhane A, Mahara D. Primary non-Hodgkin's lymphoma of bone arising from hip. J Nepal Health Res Counc. 2013;11(23):89-91.

32. McCammon J Mascarenhas $R$, Monument MJ, Elyousfi A, Pilkey B Large B-cell lymphoma mimicking iliopsoas abscess following open revision of proximal femur infected non-union: A case report. BMC Res Notes. 2014;7(1):1-5.

33. Misgeld E, Wehmeier A, Krömeke O, Gattermann N. Primary non-Hodg kin's lymphoma of bone: Three cases and a short review of the literature. Ann Hematol. 2003;82(7):440-3.

34. Pinheiro R, Filho F, Lima G, Ferreira F. Primary non-Hodgkin lymphoma of bone: An unusual presentation. J Cancer Res Ther. 2009;5(1):52-3.

35. Riaz I Bin, Khan MS, Mazursky K, Husnain M, Anwer F. Bone lymphoma with multiple negative bone biopsies. J Am Acad Physician Assist. 2017;30(9):27-9

36. Romero-Rojas AE, Diaz-Perez JA, Raju S, Messa-Botero O, Prieto-Ble$\tan A$, Criollo-Palacios F. Primary diffuse large B-cell lymphoma associated with chronic osteomyelitis of the knee. Knee. 2014;21(6):1280-3.

37. Roy S, Dobson P, Henry L. An isolated osteochondroma with underlying non-Hodgkin's lymphoma of bone. J Bone Joint Surg Am. 2005;87(10):1423-4

38. Sato H, Takahashi M. Non-hodgkin's malignant lymphoma of the bone with intracavitary cardiac involvement. Intern Med. 1993;32(6):502-7.

39. Schmidt AG, Kohn D, Bernhards J, Braitinger S. Solitary skeletal lesions as primary manifestations of non-Hodgkin's lymphoma. Arch Orthop Trauma Surg. 1994;113(3):121-8.

40. Schulze T, Lüdtke A, Rahlff I, Tunn PU, Hohenberger P. Salmonella osteomyelitis in an immunocompromized patient presenting as a primary lymphoma of the bone. Int J Infect Dis. 2009;13(2):36-9.
41. Singh T, Satheesh C, Lakshmaiah K, Suresh T, Babu G, Lokanatha D, et al. Primary bone lymphoma: A report of two cases and review of the literature. J Cancer Res Ther. 2010;6(3):296-8.

42. Singh $\mathrm{R}$, Al Wattar $\mathrm{BH}$, Mohanty K. A rare case of primary bone lymphoma mimicking a pelvic abscess. Ann R Coll Surg Engl. 2011;93(7):142-4.

43. Sipsas NV, Kontos A, Panayiotakopoulos GD, Androulaki A, Zormpala A, Balafouta ME, et al. Extranodal non-Hodgkin lymphoma presenting as a soft tissue mass in the proximal femur in a HIV (+) patient. Leuk Lymphoma. 2002;43(12):2405-7.

44. Stephens GC, Lennington WJ, Schwartz HS. Primary lymphoma and Paget's disease of the femur. Am J Clin Pathol. 1994:101(6):783-6.

45. Sugisawa N, Suzuki T, Hiroi N, Yamane T, Natori K, Kiguchi H, et al. Primary bone malignant lymphoma: Radiographic and magnetic resonance images. Intern Med. 2006;45(9):665-6.

46. Visser J, Busch VJ, de Kievit-van der Heijden IM, Ten Ham AM. Non-Hodgkin's Lymphoma of the synovium discovered in total knee arthroplasty: A case report. BMC Res Notes. 2012;5:5-8.

47. Xie XT, Gao YS, Zhang CQ. Salvage of a femoral nonunion after primary non-Hodgkin's lymphoma of bone: A case report and literature review. Med Sci Monit. 2011;17(11):138-43.

48. Yamamoto S, Tanaka T, Sato K, Denda R, Kumagae $Y$, Sekiya H, et al. Primary non-germinal center B-cell-like diffuse large B-cell lymphoma arising from the patella. J Orthop Sci. 2019;24(3):568-72.

49. Böhm P, Kunz W, Horny HP, Einsele H. Adult Gaucher dissease in association with primary malignant bone tumors. Cancer. 2001:91(3):457-62.

50. Ngan H, Preston BJ. Lymphoma presenting with osseous lesions. Clin Radiol. 1975;26:351-6.

51. Radaszkiewicz T, Hansmann ML. Primary high-grade malignant lymphomas of bone. Virchows Arch A Pathol Anat Histopathol. 1988:413(4):269-74.

52. Sweet DL, Mass DP, Simon MA, Shapiro CM. Histiocytic lymphoma (reticulum-cell sarcoma) of bone. J Bone Joint Surg Am. 1981; 63(1):79-84.

53. Lucraft $\mathrm{HH}$. Primary limphoma of bone: A review of 13 cases emphatizing orthopaedic problems. Clin Oncol. 1991;3:265-9. 